\title{
Wavefront compensation method using novel index in holographic data storage
}

\author{
Norihiko Ishii \\ ishii.n-gm@nhk.or.jp

\section{Tetsuhiko Muroi} \\ Nobuhiro Kinoshita
}

\section{Koji Kamijo}

\section{Naoki Shimidzu}

\begin{abstract}
Science \& Technology Research Laboratories, Japan Broadcasting Corporation (NHK) 10-11, Kinuta 1-chome, Setagaya-ku, Tokyo, 157-8510, Japan

Science \& Technology Research Laboratories, Japan Broadcasting Corporation (NHK) 10-11, Kinuta 1-chome, Setagaya-ku, Tokyo, 157-8510, Japan

Science \& Technology Research Laboratories, Japan Broadcasting Corporation (NHK) 10-11, Kinuta 1-chome, Setagaya-ku, Tokyo, 157-8510, Japan

Science \& Technology Research Laboratories, Japan Broadcasting Corporation (NHK) 10-11, Kinuta 1-chome, Setagaya-ku, Tokyo, 157-8510, Japan

Science \& Technology Research Laboratories, Japan Broadcasting Corporation (NHK)

10-11, Kinuta 1-chome, Setagaya-ku, Tokyo, 157-8510, Japan
\end{abstract}

Photopolymer media that uses holographic data storage generally causes volume shrinkage. This volume shrinkage distorts the recorded interference fringes. We propose an adaptive optics using novel index to compensate this distortion. The SNR value is improved from $2.1 \mathrm{~dB}$ to $3.4 \mathrm{~dB}$ in the worst part of a reproduced image and the peak intensity and the full width of the half maximum of an angle are improved by $6 \%$ and $10 \%$, respectively. These results prove that adaptive optics using this novel index worked effectively. [D0I: 10.2971/jeos.2010.10036s]

Keywords: holographic data storage, adaptive optics, wavefront compensation, photopolymer

\section{INTRODUCTION}

Since holographic data storage systems can record twodimensional bit data in the same recording area using multiplexing, high density recording and a high data-transfer-rate is possible. Photopolymers, which are a write-once type of media, are promising as holographic recording media since they have a large diffraction efficiency and are highly stable [1]. When writing data as a refractive index change using photopolymerization, this phenomenon causes volume shrinkage. Although a low-shrinkage photopolymer, which consists of 2 chemistry material [2] and a seemingly zero-shrinkage photopolymer [3] were developed, a small amount of distortion occur in the recording spot. The recorded interference fringes are distorted by these volume shrinkages, and this created in-area distribution in the twodimensional bit data and consequently bit errors occurred during reproduction.

On the other hand, in digital holography and threedimensional display, the deformed hologram is also serious problem, especially the depth of focus. Several approaches are proposed [4]. Recently numerical transformation method was proposed and it can be applied to Fourier-type holograms and thereby physically recorded on a photosensitive media like photopolymer $[5,6]$.

We propose another approach that compensates the distorted fringes, which controls the wavefront of the reference beam and obtains a high-quality reproduced image. We have previously reported that the wavefront was controlled to raise the evaluation index based on the Signal-to-Noise Ratio (SNR) using a genetic algorithm (GA) and that the SNR could be improved [7]. However, since the SNR must be evaluated using the entire reproduced image, a lot of calculation time is needed. Accordingly, a block SNR, which is calculated in the small divided area of page, is proposed to reduce the calculation time. However, since statistics values such as the mean value and standard deviation are used, the reliability is lower because there are not many pixels.

In this paper, a novel index of page data is proposed instead of a block SNR. This index was used to compensate for the uniformity of a reproduced image caused by media shrinkage, and we confirmed the effectiveness of this method.

\section{THEORY OF FRING MICRO-DISTORTION}

Figure 1 shows the configuration of the Bragg diffraction on a k-sphere in a holographic medium.

Figure 1(a) is the ideal recording and reproduction conditions. In this figure, the signal beam vector, reference beam vector, and recorded vector are $\mathbf{k}_{\mathrm{s}}, \mathbf{k}_{\mathrm{rw}}$, and $\mathbf{K}$ respectively. In the recording procedure, $\mathbf{k}_{\mathrm{s}}$ and $\mathbf{k}_{\mathrm{rw}}$ are irradiated and fringe vector $\mathbf{K}$ is generated in the recording media. In the reproducing procedure, $\mathbf{k}_{\mathrm{rw}}$ is irradiated and the signal beam $\mathbf{k}_{\mathrm{s}}$ is diffracted by the fringe vector $\mathbf{K}$ [8]. However, when photopolymer is used as the recording medium, volume 


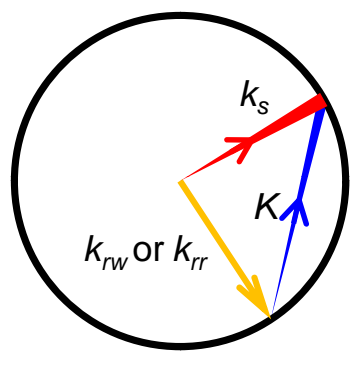

(a)

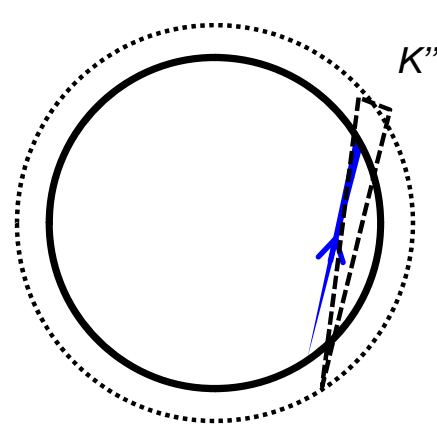

(c)

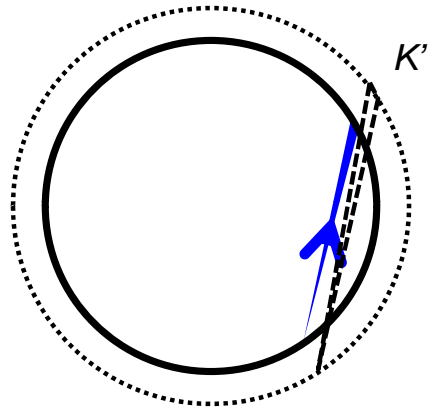

(b)

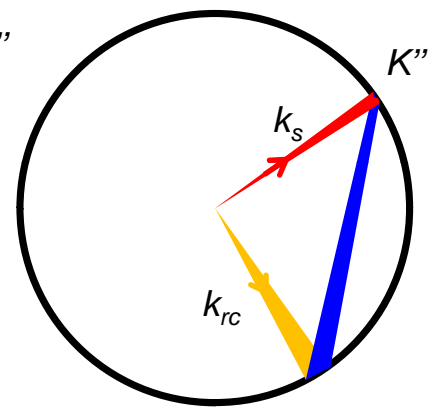

(d)
FIG. 1 K-Sphere under given conditions. (a) Ideal condition of hologram; (b) isotropic shrinkage condition; (c) anisotropic shrinkage condition; and (d) proposed compensation method.

shrinkage generally occurs. In addition, volume expansion or shrinkage of the photopolymer occurs due to ambient temperature changes during reproduction. The recorded fringes also expand or shrink and change to different conditions. If these changes happen, images cannot be reproduced due to unsatisfactory diffraction conditions.

Figure 1(b) shows a photopolymer that shrunk isotropically, and Figure 1(c) shows a photopolymer that shrunk anisotropically. As the Bragg selectivity shifts only in the angle direction because the entire media changes isotropically in the case presented in Figure 1(b), the Bragg condition can be satisfied by tuning the angle between the reference beam and the photopolymer. Thus, the image can be reproduced. In anisotropic volume shrinkage, the recorded vector $\mathbf{K}$ spreads and a combination of many kinds of vectors since $\mathbf{K}$ changes due to the position. Although a method, which compensate for the change in angle due to the change in wavelength of the laser source, have been proposed in isotropic shrinkage [9], it cannot compensate for the in-plane distribution caused by anisotropic shrinkage.

We proposed a method that the reference beam having compensated wavefront was irradiated. Figure 1(d) shows the compensation condition on $\mathbf{k}$-sphere. Vector $\mathbf{k}_{\mathrm{rc}}$, which is the reference beam vector, is adjusted depending on the distorted $\mathbf{K}^{\prime \prime}$ to reproduce all the $\mathbf{k}_{\mathrm{s}}$. It is important to accurately produce the wavefront since there are many kinds of vectors, as shown in the figure.

\section{NOVEL INDEX FOR COMPENSATION OF REPRODUCED DATA}

\subsection{Current Index}

The detection of the distortion of the fringes is important since the compensated reference beam can compensate for the reproduced image as described in Section 2. We tried to use a feedback control and an optimization method using the SNR as an evaluation index. The SNR in holographic data storage is represented by

$$
\mathrm{SNR}=20 \log \frac{\mu_{1}-\mu_{0}}{\sqrt{\sigma_{1}^{2}+\sigma_{0}^{2}}},
$$

where $\mu_{1}$ and $\mu_{0}$ represent the mean brightness of the reproduced bit data " 1 " and " 0 ", respectively, and $\sigma_{1}$ and $\sigma_{0}$ are the standard deviations of the reproduced bit data " 1 " and " 0 " respectively [10].

Since the relationship between media shrinkage and input wavefront is a non-linear response, the feedback control method couldn't make the optimized wavefront. In an optimization method using the SNR, the reconstructed image converged to a darkened one from which data could not be reproduced although SNR values increased.

We solved these problems by using the $\sigma$ normalized by $\mu$ as expressed in Eq. (2) and a genetic algorithm that is a kind of optimization method [7].

$$
\text { index }_{\mathrm{SNR}}=\frac{\mu_{1}-\mu_{0}}{\sqrt{c_{1}^{2}+c_{0}^{2}}} .
$$

Here, $c_{n}(n=0,1)$ is $c=\sigma_{n} / \mu_{n}$. However, a lot of calculation time is needed to evaluate the entire image using this method. Accordingly, a block SNR, which is calculated in the small divided area of page, is proposed to reduce the calculation time. However, since statistics values such as the mean value and standard deviation are used, the reliability is lower because there are not many pixels. We investigated the novel index instead of the index $x_{\text {SNR }}$.

\subsection{Novel Index}

A bar code or squared synchronized pattern is usually superimposed to align the reproduced image in a holographic data storage system [11]. A novel pattern, which can indicate the index, is used instead. The signal intensity and signal distortion are expressed in the numerator and denominator of Eq. (1) respectively. So, the single frequency pattern was used as the novel index. We used the optical setup in Figure 2 to clarify the relationship between the SNR and signal, which has a single frequency. A Spatial Light Modulator (SLM) was used to modulate the laser beam to random signals or single frequency signals, as shown in Figure 3, and modulated beam passed through the two lenses and was detected by a Charge Coupled Device (CCD) camera.

The SNR was calculated in Eq. (1) for random data, and the Fast Fourier Transform (FFT) was calculated and was used to pick up the amplitude of the fundamental frequency $A\left(f_{1}\right)$ 


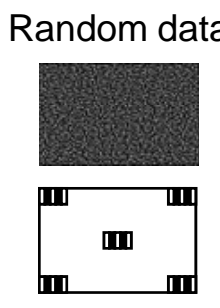

Sync pattern

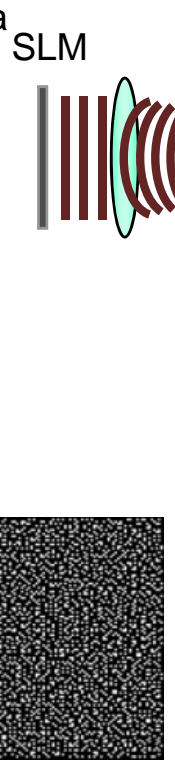

(a) Random data

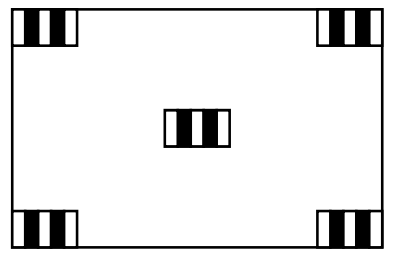

(b) Sync pattern

FIG. 3 Input image.

and the amplitude of the second harmonic frequency $A\left(f_{2}\right)$ for a single frequency pattern with changing CCD position. These results are shown in Figure 4. All of the measured data show the large dependency on the CCD position. The main reason why this dependency is apparent is because of the distortion due to defocusing. $A\left(f_{1}\right)$ and the SNR have different CCD positions in cases where the maximum amount of data is presented. We found that $A\left(f_{1}\right)$ shows that the amplitude of a signal cannot express the SNR value. We defined the SpaceFrequency Amplitude-to-distortion Ratio (SFAR) as follows:

$$
\mathrm{SFAR}=\frac{A\left(f_{1}\right)}{A\left(f_{2}\right)}
$$

Since SFAR is the signal amplitude $A\left(f_{1}\right)$ divided by the signal distortion $A\left(f_{2}\right)$, it is in good agreement with the SNR. In other words, SFAR can be used instead of SNR. The merits of using the SFAR are the low amount of noise influence using FFT, high sensitivity, fast calculation time, and that it occupies a small area.

\section{EXPERIMENTAL RESULTS AND DISCUSSIONS}

\subsection{Optical setup and method of genetic algorithm}

The optical compensation of a distorted reproduced image caused by a photopolymer used as holographic recording media was examined using this SFAR index. The optical configuration is shown in Figure 5 and the experimental conditions are described in Table 1 . The laser beam was aligned by using a spatial filter and then was magnified. A Polarized Beam Splitter (PBS) divides a laser beam into the reference and object beams. The object beam is irradiated to the SLM after the PBS. The SLM generated the pattern to rotate the polarization and only these areas could be reflected in the PBS.

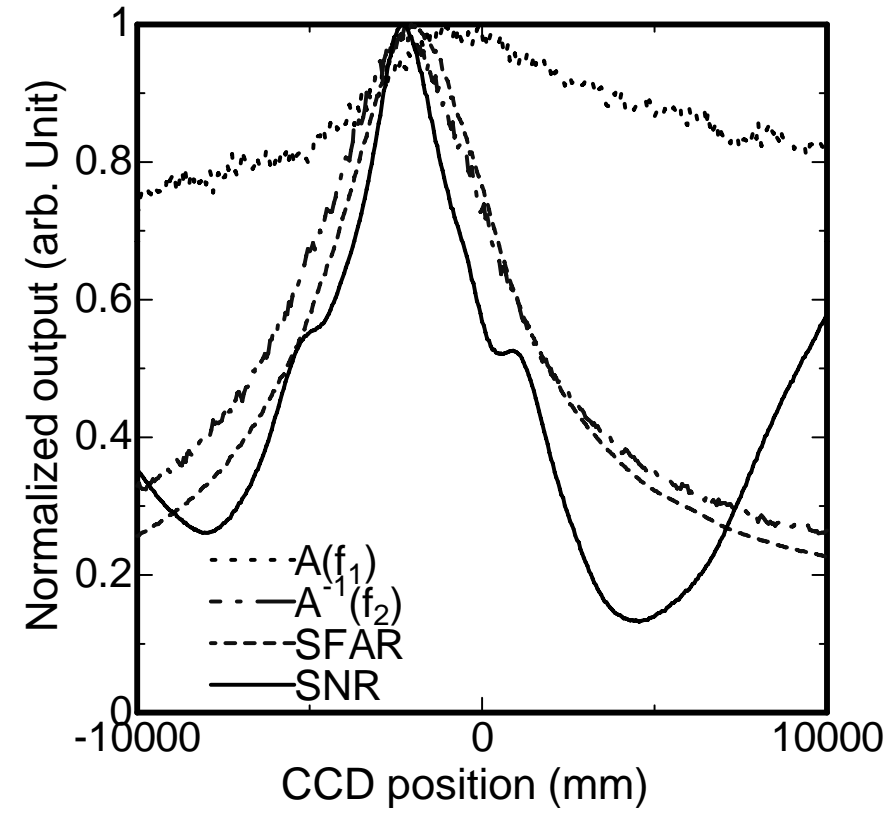

FIG. 4 FFT signals, SFAR, and SNR as function of CCD position.

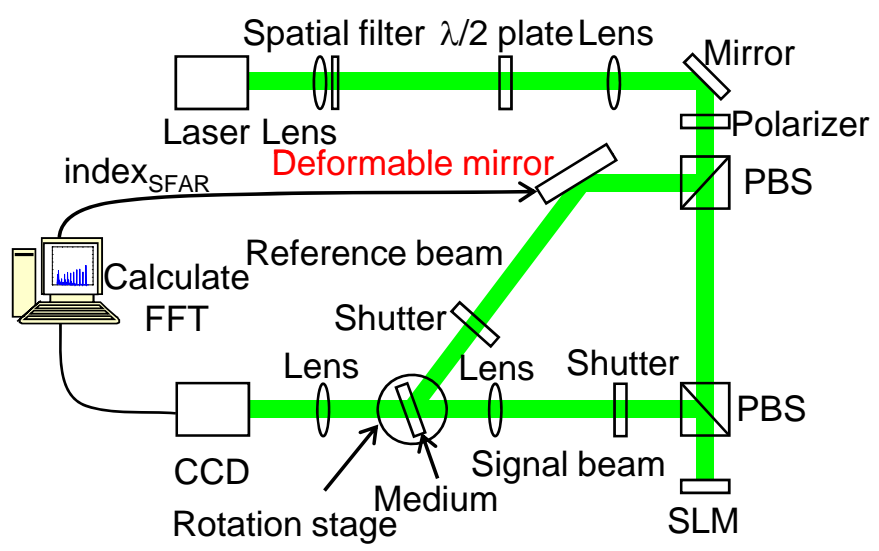

FIG. 5 Optical configuration.

\begin{tabular}{|c|c|}
\hline $\begin{array}{c}\text { Wavelength of recording } \\
\text { and reproduction }\end{array}$ & $532 \mathrm{~nm}$ \\
\hline Focal length of objective lens & $92 \mathrm{~mm}$ \\
\hline NA of objective lens & 0.033 \\
\hline SLM & $1408 \times 1058$ \\
\hline 1 block & 9 pixels $\times 9$ pixels \\
\hline Deformable mirror (diameter) & $50 \mathrm{~mm}$ \\
\hline No. of pins & 19 \\
\hline Actuator type & Piezo actuator \\
\hline Recording medium & $\begin{array}{c}\text { Photopolymer M-110 } \\
\text { and M-1600 (supplied } \\
\text { by Toagosei Co., Ltd.) }\end{array}$ \\
\hline Estimated shrinkage ratio [1] & $0.2 \%$ \\
\hline
\end{tabular}

TABLE 1 Experimental conditions.

On the other hand, the reference beam was reflected at the first PBS and was incident to the recording media after reflecting off the deformable mirror, which can control the wavefront. The surface of the deformable mirror was flat in the recording procedure; it was only controlled to fit the media shrinkage in the reproduced procedure. 


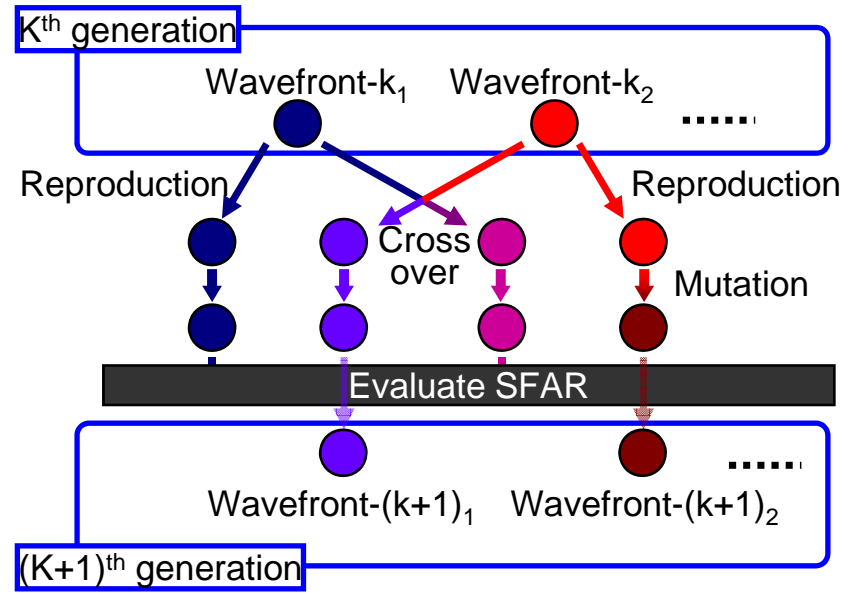

FIG. 6 Genetic algorithm.

\begin{tabular}{|c|c|}
\hline No. of individuals in population & 50 \\
\hline Generation alternation & 50 \\
\hline Mutation probability & 0.1 \\
\hline
\end{tabular}

TABLE 2 Experimental conditions of genetic algorithm.

A Genetic Algorithm (GA) [7], which is a kind of optimization method, was used for the wavefront control method. A flow chart of the GA is shown in Figure 6, and the parameters used in the GA are listed in Table 2. The parameters used in the GA are the genes, individuals, population, and fitness. The genes are defined as the values of the pins driving the DM. The individual is the condition of the DM, as determined by the genes. The population is a group of $s$ individuals. The fitness is an evaluation parameter, which represents how well the individual fits the environment. We initially have a $k^{\text {th }}$ generation population $P_{k}$, which consists of $s$ individuals. Each value for the DM's pins of the individuals in the initial population $P_{0}$ is randomly determined. The first operations are the reproduction and crossover. In the crossover, two individuals are selected from the population, and a crossover point is randomly defined.

For example, consider a crossover for individual $X$ and $Y$, and the crossover point is between pin numbers $p$ and $p+1$. The values for pin numbers less than $p$ for individual $X$ and for pin numbers greater than $p+1$ for individual $Y$ are mixed, resulting in an individual $X^{\prime}$. Likewise, the value for the pin numbers greater than $p+1$ for individual $X$ and for pin numbers less than $p$ for individual $Y$ are mixed, resulting in an individual $Y_{0}$. As a result, two new individuals were generated. This crossover is applied to all individuals, and the resulting population $P_{k}^{\prime}$ consists of $2 s$ individuals made by reproduction and crossover.

The second operation is mutation. An individual, except the one with the highest fitness value in population $P_{k-1}$, is selected with a probability $F m$ from $P_{k 0}$. The value of the randomly selected pin is randomly changed for the selected individual. After that, for each individual, the DM values are set, the reconstructed image is measured with a CCD, and the fitness is calculated. Finally, $s$ individuals with higher fitness values are selected, and they form the nextgeneration popula-

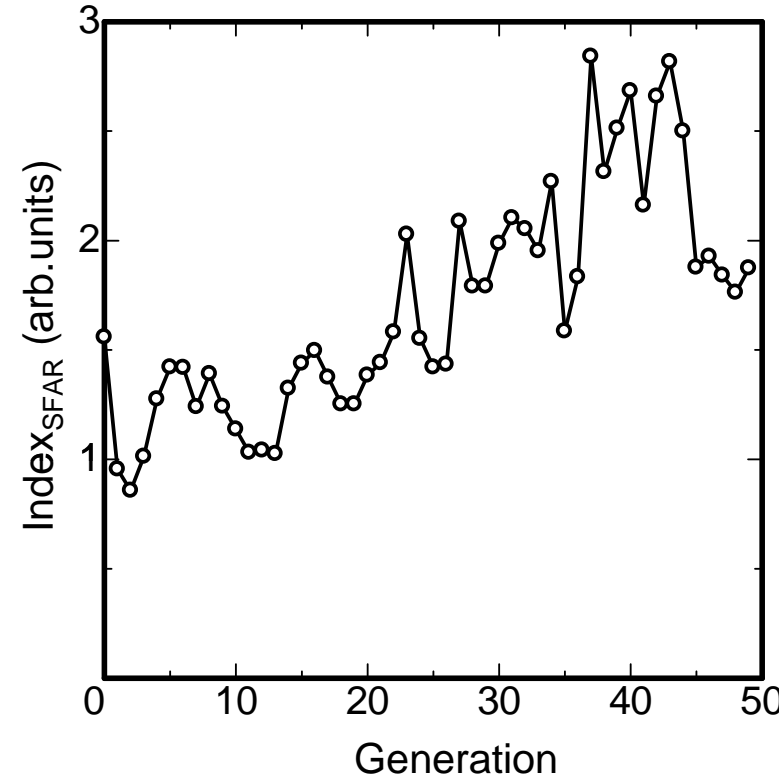

FIG. 7 Index $x_{S F A R}$ build up.

tion $P_{k+1}$. The optimized wavefront is obtained by repeating this process until the fitness value saturates.

\subsection{Index of SFAR build up and reproduced image}

The patterns of a single space frequency were superimposed to each corner and the center and the SFAR average was calculated to become a larger value using the wavefront compensation method described in the former section.

Figure 7 shows the relationship between the SFAR index and the generation alternation. Good and bad indexes are alternately shown, but the control trend is good on the whole. Even though a wavefront remained for the next generation in the generation alternation, there is a case in which a lower index than that in the previous generation is shown. The reason for this is a fluctuation in the measured data. The reproduced image fluctuates due to air turbulence and a small vibration [12]. Furthermore, the thermal noise or shot noise of the CCD can be thought.

The reproduced images of the initial situation and the $50^{\text {th }}$ generation are shown in Figure 8. Figures 8(a) and 8(b) show the before and after compensation of the reproduced image, respectively. In particular, the image surrounded by the red line is darker due to the Bragg mismatch in Figure 8(a), but, the signal can be read clearly from there in Figure 8(b). The SNR values in that area are $2.1 \mathrm{~dB}$ in Figure $8(\mathrm{a})$ and $3.4 \mathrm{~dB}$ in Figure $8(\mathrm{~b})$ respectively and a $1.3 \mathrm{~dB}$ SNR improvement can be obtained.

\subsection{Bragg selectivity of reproduced image before and after compensation}

Six angle multiplexing recordings from $0^{\circ}$ to $-6^{\circ}$ of a $1^{\circ} \mathrm{du}-$ ration were examined. Figure 9 shows the sum of the reproduced image while rotating the recording medium using a 


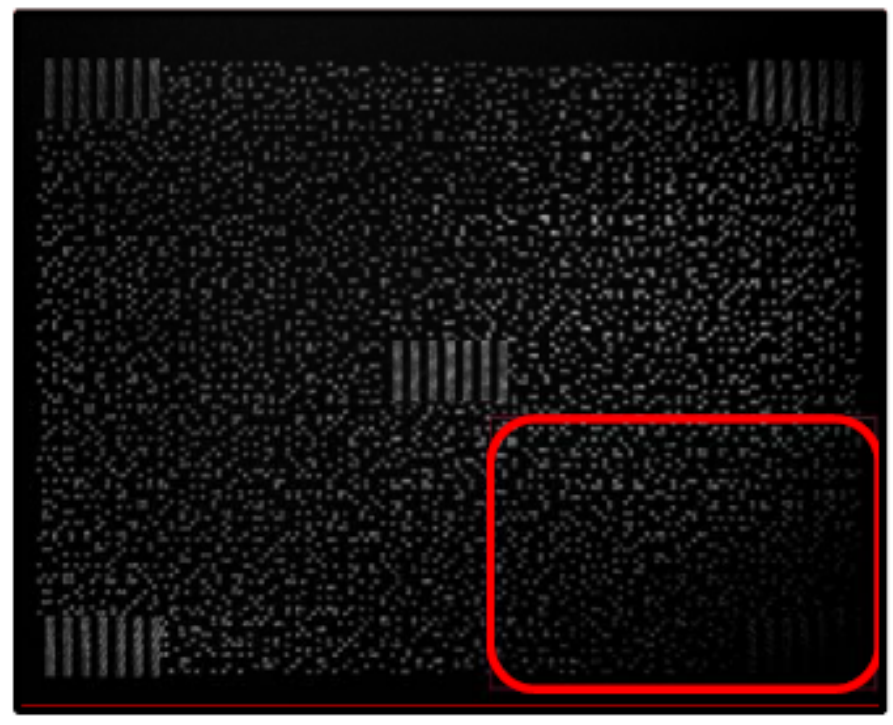

(a) Before compensation

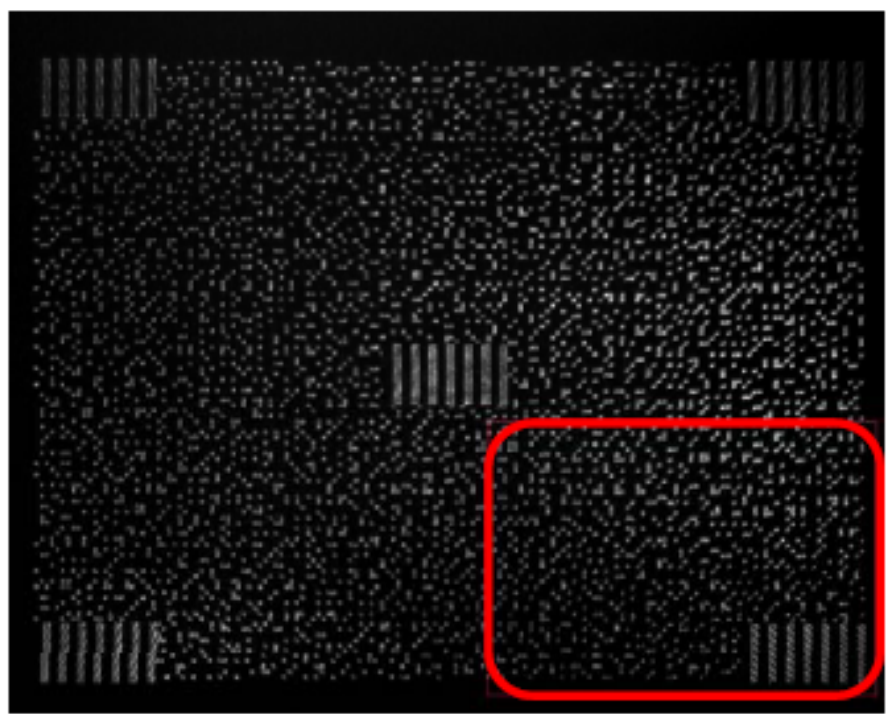

(b) After compensation

FIG. 8 Reproduced image.

flat wavefront corresponding to the recording wavefront and using an optimized wavefront for the $1^{\text {st }}$ page, which is controlled by the method described in Section 4.2. The page numbers are shown near peak in this figure. Photopolymer shrinks due to irradiation upon recording and post irradiation to fix the remaining active monomer, and all the reproduced peaks are in different positions compared with the recording positions from the $1^{\text {st }}$ to $6^{\text {th }}$ page. The main reason for the Bragg detune is due to the isotropic shrinkage described in Section 2. In addition, the phenomena where the largest peak is on page 2 and where the peak intensity decreases with increasing number of pages are caused by the sensibility of the recording medium. The sensibility of the photopolymer against cumulative power is usually shown as an s curve, which consists of a rising part, a linear part, and an ending part. So, the second peak represents the largest peak intensity and the peaks after the second one decrease the intensity as the number of pages increases.

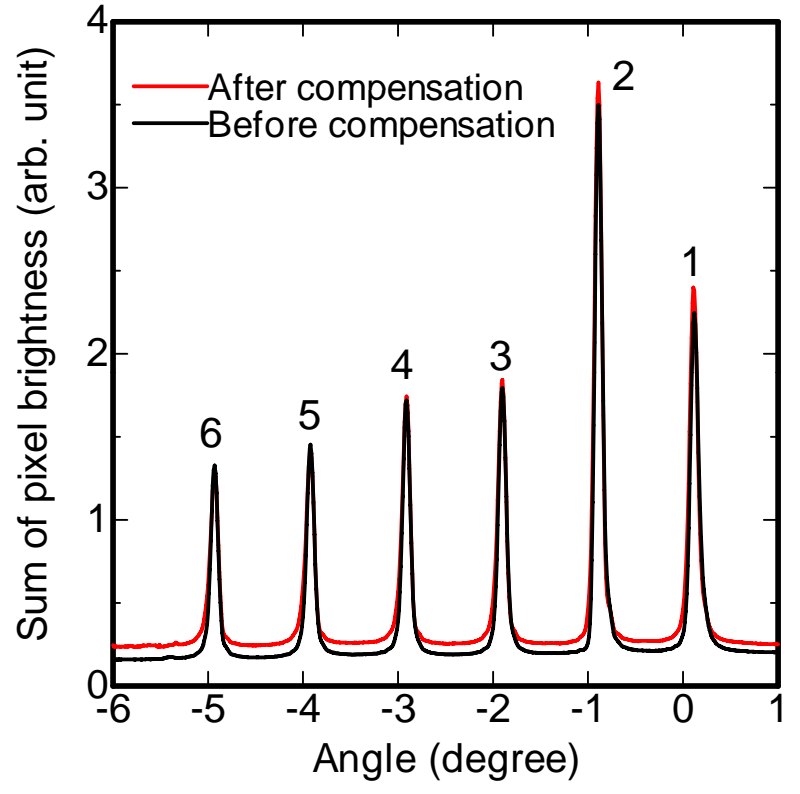

FIG. 9 Bragg selectivity.

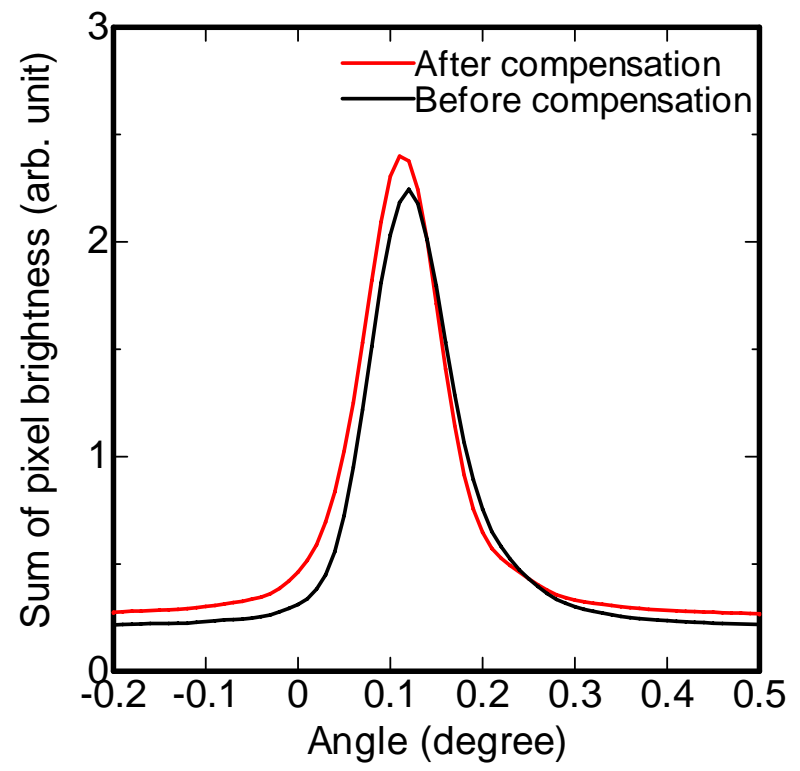

FIG. 10 Bragg selectivity for page 1 .

The optimized wavefront for page 1 can compensate for the $1^{\text {st }}$ to $4^{\text {th }}$ pages, the optimized wavefront for each page can be predicted as a gradual change. The Zoom figure of Figure 9 around page 1 is shown in Figure 10. The peak intensity and Full-Width Half Maximum (FWHM) after compensation are larger and narrower than that of the non-compensated one, respectively. The peak intensity can be improved from $2.25 \times 10^{8}$ to $2.40 \times 10^{8}$, which corresponds to a $6 \%$ improvement and the FWHM can be improved from $10.3 \times 10^{-2}$ to $9.9 \times 10^{-2}$, which corresponds to a $10 \%$ improvement. This $10 \%$ improvement of the FWHM can contribute to high multiplexing recording because of possible of the narrow angle pitch.

\section{CONCLUSION}

In holographic data storage, photopolymerization while recording data causes the photopolymer material used in 
the medium to shrink. Shrinkage distorts the recorded interference fringes and degrades the SNR of the reproduced two-dimensional discrete data. We developed a method for compensating for this distortion by controlling the wavefront of the reference beam and a novel index is proposed for this control. This novel index is similar to the SNR because the signal intensity is divided by signal distortion, and it can be used instead of the SNR. The part of the reproduced image that is the most degraded can be improved from $2.1 \mathrm{~dB}$ before compensation to $3.4 \mathrm{~dB}$ after compensation; and the $1.3 \mathrm{~dB}$ improvement can be obtained when we apply the wavefront compensation method using this novel index. In addition, the peak intensity and FWHM of the Bragg selectivity can also be improved. These results mean that this novel index worked effectively and the wavefront compensation succeeded.

\section{References}

[1] L. Dhar, M. Schnoes, T. Wysocki, H. Bair, M. Schnoes, and C. Boyd, "Temperature-induced changes in photopolymer volume holograms" Appl. Phys. Lett. 73, 1337-1339 (1998).

[2] L. Dhar, A. Hale, H. Katz, M. Schilling, M. Schnoes, and F. Schilling, "Recording media that exhibit high dynamic range for digital holographic data storage" Opt. Lett. 24, 487-489 (1999).

[3] Y. Usami, T. Sasaki, M. Kamo, S. Yamada, H. Suzuki, and M. Yumoto, "Low angular distortion due to shrinkage after fixing in new holographic recording material" Proc. SPIE 6620, 66201 H-66201 H-8 (2007).

[4] A. Zlotnik, S. Ben-Yaish, and Z. Zalevsky, "Extending the depth of focus for enhanced three-dimensional imaging and profilometry: an overview" Appl. Opt. 48, H105-H112 (2009).

[5] P. Ferraro, M. Paturzo, P. Memmolo, and A. Finizio, "Controlling depth of focus in $3 \mathrm{D}$ image reconstructions by flexible and adaptive deformation of digital holograms" 0 pt. Lett. 34, 2787-2780 (2009).

[6] M. Paturzo, and P. Ferraro, "Creating an extended focus image of a tilted object in Fourier digital holography" Opt. Express 17, 20546-20552 (2009).

[7] T. Muroi, N. Kinoshita, N. Ishii, K. Kamijo, and N. Shimidzu, “Optical compensation of distorted data image caused by interference fringe distortion in holographic data storage" Appl. Opt. 48, 36813690 (2009).

[8] G. Barbastathis, and D. Psaltis, "Volume holographic multiplexing methods" in Holographic Data Storage, H. Coufal, D. Psaltis, and G. Sincerbox, eds., 21-62 (Springer-Verlag, Berlin, 2000).

[9] M. Toishi, T. Tanaka, M. Sugiki, and K. Watanabe, "Improvement in temperature tolerance of holographic data storage using wavelength tunable laser" Jpn. J. Appl. Phys. 45, 1297-1304 (2006).

[10] G. W. Burr, H. Coufal, R. K. Grygier, J. A. Hoffnagle, and C. M. Jefferson, "Noise reduction of page-oriented data storage by inverse filtering during recording" Opt. Lett. 23, 289-291 (1998).

[11] D. Woods, and K. Malang, "Micro-positioning movement of holographic data storage system components" U. S. Patent US7116626 (2006).

[12] N. Ishii, N. Kinoshita, T. Muroi, K. Kamijo, and N. Shimidzu, "Method of phase compensation for holographic data storage" Jpn. J. Appl. Phys. 46, 3862-3866 (2007). 